Article

\title{
Encouraging Sustainable Transport Choices in American Households: Results from an Empirically Grounded Agent-Based Model
}

\section{Davide Natalini $^{1, *}$ and Giangiacomo Bravo ${ }^{2}$}

1 Global Sustainability Institute, Anglia Ruskin University, East Road, Cambridge CB1 1PT, UK

2 Department of Social Studies, Linnaeus University, and Collegio Carlo Alberto, Universitetsplatsen 1, 35252 Växjö, Sweden; E-Mail: giangiacomo.bravo@lnu.se

* Author to whom correspondence should be addressed; E-Mail: davide.natalini@anglia.ac.uk; Tel.: +44-845-196-5115.

Received: 3 September 2013; in revised form: 5 December 2013 / Accepted: 9 December 2013 / Published: 20 December 2013

\begin{abstract}
The transport sector needs to go through an extended process of decarbonisation to counter the threat of climate change. Unfortunately, the International Energy Agency forecasts an enormous growth in the number of cars and greenhouse gas emissions by 2050. Two issues can thus be identified: (1) the need for a new methodology that could evaluate the policy performances ex-ante and (2) the need for more effective policies. To help address these issues, we developed an Agent-Based Model called Mobility USA aimed at: (1) testing whether this could be an effective approach in analysing ex-ante policy implementation in the transport sector; and (2) evaluating the effects of alternative policy scenarios on commuting behaviours in the USA. Particularly, we tested the effects of two sets of policies, namely market-based and preference-change ones. The model results suggest that this type of agent-based approach will provide a useful tool for testing policy interventions and their effectiveness.
\end{abstract}

Keywords: Agent-Based Model; environmental policies; price-based policies; preference-based policies; sustainability; transports

\section{Introduction}

During the last few decades, many policies have been implemented to address the ever-growing emission trends generated by the transport sector. Notwithstanding the steps taken in promoting more 
sustainable travels, the sector still accounts for $22 \%$ of global $\mathrm{CO}_{2}$ emissions [1] and up to $50 \%$ in cities [2]. In addition to that, the International Energy Agency (IEA) forecasts an increase of $250 \%-375 \%$ in the number of cars by 2050 [3]. Correspondingly, $\mathrm{CO}_{2}$ emissions from this sector are projected to experience a $300 \%$ growth in the same period [3]. Note that the latter figure is almost five times higher than the reduction recommended by the IEA to meet Intergovernmental Panel on Climate Change's (IPCC) target of avoiding catastrophic climate change [4].

The attention given to the transport sector for its potential contribution to the reduction in global $\mathrm{CO}_{2}$ emissions led to a proliferation of policies for sustainable transports. These can be grouped in three broad categories [5-9]: (1) market-based policies; (2) structural policies; and (3) preference-change policies. The first category comprises all the price-related policies, e.g., carbon, fuel or kilometre taxes, cap and trade schemes, subsidies to public transport, increase in parking charges, motor vehicle fuel economy labelling, fuel efficiency and renewable fuel standards. The second category includes policies aimed at promoting investments in infrastructures and sustainable practices, e.g., development of plans for mixed vehicle uses, build and improve cycle lanes, provide cycle parking, wider, better maintained and cleaner pavements, improved street furniture, safer neighbourhoods and crossings and promoting investments for the development and adoption of alternative fuels. The third category comprises policies directly aimed at changing people's preferences, such as advertising and marketing campaigns, education in sustainable practices, eco-driving campaigns and knowledge transfer.

Despite the widely recognised role of technology in the abatement of $\mathrm{CO}_{2}$ emissions from the transport sector (e.g., through the spreading of low-emission vehicles), it is difficult to foresee a quick shift to a zero- or, at least, a low-emission fleet. In fact, the implementation of more aggressive technology-oriented policies is likely to be difficult and would involve strong equity issues [10]. Thus, the policy-making field should also focus on policies that could facilitate a shift to sustainable behaviours. However, it is clear that no "panacea" exists, i.e., a single type of governance system able to succeed in all or, at least, in most cases [11].

Given the impressive amount of policies being developed in the transport field, and because no panacea looks possible, there is an increasing need for new methodologies that could assess ex-ante (i.e., before the implementation of the policy itself) their performance. To address this issue, we created the Mobility-USA (MUSA) model, an Agent-Based Model (ABM) able to test the implementation of policies in the transport sector, and we analysed the response of a sample of American commuters to a set of policies aimed at reducing private car use. Our focus on the USA is for three main reasons: (1) the overall impact of the country's transport system on the global climate; (2) the low price of oil in the country, which contributes to a large share of private-motor-vehicle transportation; (3) the availability of reliable data.

We focussed on commuters because they are an important target for policy-making due to their systematic and customary nature. According to the 2009 National Household Travel Survey (NHTS), the percentage of commutes by mode of transport in the USA is: $91.4 \%$ by private vehicles, $3.7 \%$ public transit, $3 \%$ walk, $1.9 \%$ other [12]. This overview of the commuting sector in the USA highlights a clear need for sound reform. Indeed, the implementation of sound and effective policies to stimulate the use of public and non-motorised transport - consequently encouraging a decrease in the share of private motorised transportation - could improve mobility options, reduce energy use and decrease Greenhouse Gases (GHG) emissions from the transport sector [13]. 
To test our modelling approach we analysed two groups of policies: market-based policies and preference-change policies. These have been chosen because they could be easily applied to our model and because of their common implementation in the transport sector [14]. The former aim at internalising the externalities involved in the production and consumption of a given good/service through the imposition of a tax/fee, whereas the latter are meant to change consumers' behaviour by acting directly upon their preferences.

The existing literature is inconclusive in showing which of these two types of policy is more effective in encouraging sustainable transport behaviours. On one hand, some authors argue that economic incentives and structural changes are more powerful than environmental knowledge in supporting environmentally-oriented behaviours [15-18]. Others go further saying that soft-policies are not likely to achieve considerable reductions in the $\mathrm{CO}_{2}$ emissions from the transport sector [6]. On the other hand, many scholars encourage an approach that indirectly affects people's behaviours through preferences and changes in attitudes. For instance, some authors proposed environmental education as a possible measure to shape more sustainable life-styles [8,19]. Conversely, Jackson [20] argues that information campaigns are less effective than other forms of learning in promoting green behaviours and Dobson [21] considers market-based instruments and social learning as being complementary instruments. Accordingly, Flachsland et al. [14] suggests that non-market policies become of pivotal importance when market failures neutralise the impacts of market-based ones. The combination of these policies is seen as an optimal strategy by different authors (e.g., [6]). On the other hand, other authors argue that information and education policies are necessary, but are not sufficient to bring about changes in behaviour [22].

The potential outcome of these policies is evaluated using the MUSA [23] model on that incorporates data derived from a sample of commuters drawn from the 2009 NHTS. In particular, we evaluate which scenario (i.e., policies implemented singularly or combined) is more effective in promoting the use of less polluting means of transport.

In order to ease the understanding of the policies being implemented in MUSA (i.e., market-based and preference-change), we provide some examples: the first type of policy could be a carbon tax that is meant to affect the use of motorised transports by increasing their price of use. The second type of policy could be imagined both as a combination of policies such as marketing campaigns, provision of information about the commuting alternatives of the agents, etc., or as a single and effective policy, such as the introduction of classes on environmental education through formal compulsory education.

The remainder of this paper is organised as follows: Section 2 defines the model following the Overview, Design concepts, Details (ODD) Protocol and provides the data on which the model relies. Section 3 grounds the model into empirical data and Section 4 presents the outcomes resulting from the policy scenarios. Finally, Section 5 presents the conclusions.

\section{Methods}

The analysis below is centred on an agent-based model, i.e., "a computational method that enables a researcher to create, analyse, and experiment with models composed of agents that interact within an environment" [24]. This definition highlights four elements that uniquely characterise ABMs: (1) an environment, i.e., a set of objects the agents can interact with; (2) a set of interactive agents; (3) a set 
of relationships linking objects and/or agents; and (4) a set of operators that allow the interaction between the agents and the objects. In particular, ABMs implement a generative approach, which allows the investigation of social patterns using a bottom-up technique. There is a notable difference between bottom-up (e.g., ABMs) and top-down (e.g., System Dynamics) approaches: in the first case, the data gathering procedure may be time-consuming and simulations may be more complex than top-down ones. However, the resulting overall macro-picture will probably be more informative [25]. Moreover, the generative approach allows the consideration of emerging properties: a given macro-behaviour cannot be represented as a simple aggregation of several micro-behaviours. In fact, ABMs can be used to analyse the non-linearity of aggregated behaviours with respect to individual ones. This bottom-up approach can bring about unexpected outcomes, which can represent emerging properties, regularities and structures [26].

\subsection{The MUSA Model}

This section has been organised following the ODD Protocol first developed by Grimm and Railsback [27] and further improved by Grimm et al. [28] and Grimm et al. [29]. The purpose of this protocol is that of providing a sequence of steps when formalising an ABM in order to arrange all the relevant information about the model in a neat and logic way. Moreover, the implementation of such protocol is also useful to the process of replication and verification of the model when these are being carried out by people others than the authors [27-30]. Notwithstanding some limitations of the ODD protocol, e.g., the possible redundancy between the categories included, it is a very good way to increase the clarity of the model specifications $[29,30]$.

\subsubsection{Purpose}

MUSA is an ABM that aims at reproducing the transport choices of a sample of American citizens and the GHG emissions related to their daily commutes. Specifically, it was designed to test whether the $\mathrm{ABM}$ approach is suitable to investigate commuting behaviours and to evaluate ex-ante the consequences of policy implementation.

\subsubsection{Entities, State Variables and Scales}

MUSA includes only one type of agent-American commuters. The state variables of the agents are: their initial and final preference for each of the modes of transport, the importance they give to their social and personal satisfaction, the number of their neighbours, their previous and current satisfaction given by the choice of the mode of transport, the uncertainty they experience while choosing the preferred mode of transport and whether they decide to make a short or a long commute (i.e., SC or LC).

The agents' position in the 3D space corresponds to their preferences for each mode of transport (i.e., the preferences act as coordinates for the dimensions $x, y$ and $z$ ), hence there is no absolute concept of spatial scale in the model (i.e., the fact that two agents are close in the 3D space does not necessarily mean they are neighbours in the reality). 
According to their position in the 3D space, the agents are clustered in neighbourhoods. The agents that belong to the same neighbourhood are connected by links, while longer links can span across neighbourhoods. This allows social influence to be taken into account when the agents make decisions.

The second entity included in MUSA is the government. It is represented in the model in an abstract form and it acts - directly or indirectly — on the agents' preferences by implementing two types of policies: market-based and preference-change policies.

As far as the time scale is concerned, one time step corresponds to one commute for every agent. Assuming that each person commutes on average twice a day, this temporal unit could be interpreted as half a day.

\subsubsection{Process Overview and Scheduling}

MUSA considers one main process, the commuters' decision about what means of transport to travel in. The agents start with a set of preferences - one for each mode of transport - that have been assigned to them and that place them in the 3D space. Throughout the process, these are influenced by the relative price of the different means of transport, by social influence and by the intensity of the policies applied.

After the assignment of the preferences to the agents, the social network is initialised and the neighbourhoods are formed according to the closeness of the initial preferences of the agents. During the following step they decide whether to make an SC or an LC and then they decide what means of transport to use.

The agents, while performing the procedures, do not follow any particular order and the variables included in the model are synchronously updated at the end of each procedure. At the following time step the process starts all over again.

\subsubsection{Design Concepts}

- Basic principles: One of the core assumptions made in MUSA is that SCs and LCs differ for the selection of the eligible means of transport. For SCs, agents can choose whether to commute by private motorised vehicles $(\mathrm{M})$, private non-motorised vehicles $(\mathrm{NM})$ or by public transportation (PT). Conversely, for LCs the choice is limited to M and PT. This distinction is based on the assumption that long distances cannot be covered with NM (See Section 2.1.6).

Agents' decisions are based on the "consumat" framework developed by Jager [31] to explain the environmental behaviour of consumers. This was first translated into an actual model by Jager and Janssen [32] and-more recently-by Bravo et al. [33]. According to this framework, agents' decisions about the means of transport rely on a set of four possible processes. Depending on their satisfaction and uncertainty, agents can employ (1) rational deliberation, (2) imitate others' behaviour, (3) compare their own satisfaction with their neighbours' one, or (4) repeat the previously chosen behaviour (see Section 2.1.7). Agents are linked in a network following a principle of homophily in preferences. Unlike Janssen and Jager [32], who employed a small-world network, we chose to use the "social circle" structure, first introduced by Hamill and Gilbert [34], which better incorporates key aspects of large social networks such as low density, high clustering and assortativity of the connectivity degree. The network is based on the ideas of social space and distance. 
- Emergence: One of the principal features of the "consumat" model is the introduction of social influence in the agents' decisions. Hence, the patterns in the use of each means of transport are emergent in the sense that they are the result of single agents' behaviours that in turn are influenced by those of their neighbours.

- Adaptation: This is another feature of the "consumat" model. Agents can employ different deliberative processes according to the situation they find themselves in. This is particularly relevant in the policy scenarios, where agents have to adapt their decisions to different relative prices of the means of transport or to influences on their initial preferences.

- Objectives: The agents, while deciding what means of transport to commute in, try to maximise their personal and social satisfaction taking their uncertainty into account (i.e., how many times they change their minds about what means of transport to commute in) (see Section 2.1.7).

- Learning: The agents, while trying to achieve their objective, can employ several deliberative processes that compare the total level of satisfaction gained by the decision taken in the previous time step and their uncertainty.

- Sensing: Two of the deliberative processes that the agents can employ while making a decision are imitation and social comparison. In both cases they will compare their past overall satisfaction with that of their neighbours.

- Interaction: The interaction between the agents occurs through the social network they are embedded in.

- Stochasticity: Randomness is included in different parts of the model. Some randomness is introduced in the agents' locations to avoid the building of exactly the same network at every run. Moreover, it is introduced when choosing a type of commute (i.e., SC or LC) and while weighing social and personal satisfaction.

- Collectives: The agents are grouped into neighbourhoods according to their position in the 3D space, which is in turn the result of their preferences for the different means of transport and some randomness. These neighbourhoods are represented in the 3D space as clusters of agents.

- Observation: The values collected and stored at the end of every time step are the number of agents making SCs and LCs, the means of transport they decide to use, the deliberative process they employ and the $\mathrm{CO}_{2}$ emissions related to each type of commute.

\subsubsection{Initialisation}

When the simulation starts, the characteristics of each means of transports are initialised. Each of them is provided with a specific relative price that can be chosen by the user in the model interface (see Section 2.2.2), average $\mathrm{CO}_{2}$ emissions per mile and an environmental index (see Section 2.2.2). Moreover, the model sets a propensity for the type of commute, which is the likelihood of one agent to choose to make an SC or an LC (see Section 2.1.6). Then MUSA creates the agents, placing them into a network built following the procedure described in Hamill and Gilbert [34]. Agents have a set of initial preferences derived from empirical data (see Sections 2.2 and 3), which also affects their position in the 3D space. Each dimension of the space maps one of the agent's preferences. Then each agent creates links with all the other agents within a certain distance, determined by the link-distance parameter. This parameter can be chosen in the model interface and in our simulations was set to a value 
leading to a degree distribution approaching a power law, as is common in many social networks [35]. Then another procedure checks if there is any lone agent. If so, this gets linked with its closest neighbour.

Finally, the agents' uncertainty is set to zero and their total level of satisfaction is computed (see Section 2.1.7).

\subsubsection{Input Data}

In MUSA, each agent $i$ is characterised by a set of preferences $P_{i}=\left\{p_{i 1}, \ldots, p_{i m}\right\}$ where $m=3$, the set of the eligible modes of transport. Each agent maps one of the 5000 respondents selected as a subsample from the 2009 NHTS survey [36] and their preferences are derived from the answers given in the survey (see Section 2.2). The analysis of the commute length declared by the participants in the 2009 NTHS led us to define SCs as between 0 and 3 miles in length - a distance that can be easily covered walking or cycling - whereas LCs are all commutes longer than 3 miles. According to the analysis on the survey, $80 \%$ of the commutes were LCs and $20 \%$ SCs. This parameter has been used to inform the choice of the agents when they had to decide whether to make an SC or an LC. The same survey has then been used to compare the results of the calibration of MUSA (see Section 3).

Another external input concerns the relative prices of the means of transport and their average emissions. These are presented in detail in Section 2.2.

\subsubsection{Submodels}

In the consumat framework, agents have both personal and social needs and their satisfaction is affected by both dimensions [31]. Personal need satisfaction $\left(\mathrm{N}_{\mathrm{ij}}^{\mathrm{p}}\right)$ is assumed to be equal to $p_{i j}$ where $j$ is the means of transport used by agent $i$. This means that the current personal need satisfaction of agent $i$ depends on the relationship between its preferences and its transportation choice in the previous time step.

Social satisfaction can be defined as the proportion of agents in agent $i$ 's neighbourhood using the same means of transport $j$ as $i$. Agents' social need satisfaction can thus be computed as follows:

$$
N_{i j}^{S}=\frac{n_{i}^{j}}{n_{i}}
$$

where $N_{i j}^{S}$ is the social need satisfaction of agent $i$ who chose the means of transport $j, n_{i}^{j}$ is the number of agents in $i$ 's neighbourhood who chose the means of transport $j$ and $n_{i}$ is the total number of agents in the neighbourhood taking the same type of commute as agent $i$.

According to Jager [31], the total level of need satisfaction $N_{i j}^{S}$ depends on both personal and social satisfaction, along with the relative price of the different modes of transport:

$$
N_{i j}=\frac{\beta_{i} N_{i j}^{s}+\left(1-\beta_{i}\right) N_{i j}^{p}}{r_{j}}
$$

where $N_{i j}$ is the total need satisfaction of agent $i$ who chose $j$ means of transport, $\beta_{i} \in[0,1]$ is a randomly distributed agent parameter that weights personal needs against social ones and $r_{j}$ is the relative price for the $j$ means of transport (see Section 2.2.2). A low value of $\beta_{i}$ implies giving more importance to personal needs than to social ones and vice versa. 
Uncertainty is the second variable affecting the selection of the deliberation procedure. It depends on the variation of agents' satisfaction over time. Since forecasting the outcome of a given behaviour is difficult when high variability is involved, it is assumed that the higher the variability, the higher the uncertainty. Following Bravo et al. [33] and Janssen and Jager [32], uncertainty is defined as:

$$
U_{i t}=\sqrt{\left|N_{i t}-N_{i(t-1)}\right|}
$$

where $t$ is the time step at which agent $i$ has to make a choice about which deliberative process to use, $N_{i t}$ is agent $i$ 's satisfaction at time $t$ and $N_{i(t-1)}$ is agent $i$ 's satisfaction at the time $(t-1)$.

The deliberative process selection is affected by two parameters: $\tau_{\mathrm{n}}$ and $\tau_{\mathrm{u}}$, both bounded within the $[0,1]$ interval. The first one represents agents' needs, while the second refers to their tolerance towards uncertainty. Both of them can be defined by the user in the model interface. Depending on the relationship between these parameters - and thus on the relationship between agents' need satisfaction and uncertainty-different deliberative processes are selected. If $\mathrm{N}_{\mathrm{i}} \geq \tau_{\mathrm{n}}$ and $\mathrm{U}_{\mathrm{i}}>\tau_{\mathrm{u}}$ (satisfied and uncertain agents), the agent $i$ will imitate the most adopted behaviour in his neighbourhood; if $\mathrm{N}_{\mathrm{i}}<\tau_{\mathrm{n}}$ and $\mathrm{U}_{\mathrm{i}} \leq \tau_{\mathrm{u}}$ (dissatisfied and certain agents) the agent $i$ will employ rational deliberation, i.e., it will compute the satisfaction that may derive from choosing each means of transport and select the most satisfactory one; if $\mathrm{N}_{\mathrm{i}} \geq \tau_{\mathrm{n}}$ and $\mathrm{U}_{\mathrm{i}} \leq \tau_{\mathrm{u}}$ (satisfied and certain agents) the agent $i$ will simply repeat its previous decision; finally, if $\mathrm{N}_{\mathrm{i}}<\tau_{\mathrm{n}}$ and $\mathrm{U}_{\mathrm{i}}>\tau_{\mathrm{u}}$ (dissatisfied and uncertain agents) the agent $i$ will compare the satisfaction that would derive from repeating its previous choice and the one deriving from the adoption of the most common behaviour in its neighbourhood. In other words, this deliberative process will make the agent choose the means of transport that brings most satisfaction. Table 1 summarises the four deliberative processes and their characteristics. In Section 3 we will present the values we assigned to these parameters as a result of a calibration process.

Table 1. Summary of the deliberative processes [33].

\begin{tabular}{|c|c|c|c|}
\hline Satisfaction & Uncertainty & Deliberative process & Process details \\
\hline $\mathrm{N}_{\mathrm{i}} \geq \tau_{\mathrm{n}}$ & $\mathrm{U}_{\mathrm{i}}>\tau_{\mathrm{u}}$ & Imitation & $\begin{array}{l}\text { Checks the means of transport distribution in the } \\
\text { neighbourhood and adopts the modal one. In case of a tie, } \\
\text { randomly selects one of the most common means of transport. }\end{array}$ \\
\hline $\mathrm{N}_{\mathrm{i}}<\tau_{\mathrm{n}}$ & $\mathrm{U}_{\mathrm{i}} \leq \tau_{\mathrm{u}}$ & Rational deliberation & $\begin{array}{l}\text { Computes the expected satisfaction for each means of } \\
\text { transport, then chooses the one leading to the highest } \\
\text { satisfaction. In case of a tie, randomly selects one of the } \\
\text { means of transport leading to the highest satisfaction. }\end{array}$ \\
\hline $\mathrm{N}_{\mathrm{i}} \geq \tau_{\mathrm{n}}$ & $\mathrm{U}_{\mathrm{i}} \leq \tau_{\mathrm{u}}$ & Repetition & Repeats the previous choice. \\
\hline $\mathrm{N}_{\mathrm{i}}<\tau_{\mathrm{n}}$ & $\mathrm{U}_{\mathrm{i}}>\tau_{\mathrm{u}}$ & Social Comparison & $\begin{array}{l}\text { Checks which means of transport is most common in the } \\
\text { neighbourhood. In case of a tie, randomly selects one of the } \\
\text { most common means of transport. Then compares the } \\
\text { expected satisfaction of the selected means of transport with } \\
\text { the previous means of transport and selects the one leading } \\
\text { to the highest satisfaction. In case of a tie, selects one of the } \\
\text { two means of transport randomly. }\end{array}$ \\
\hline
\end{tabular}




\subsection{Empirical Foundation of MUSA}

The approach used to ground MUSA into empirical values is Multilevel Validation [25]. This has been allowed by the structure of the 2009 NHTS database, which is composed by four sub-datasets, namely Household File, Person File, Vehicle File and Travel Day Trip File. The data drawn from the Person file was used as inputs for MUSA to determine agents' preferences, while data from the Travel Day Trip file (TDTD) served as reference for the model outputs.

\subsubsection{Agents' Preferences}

In order to build the agents' preferences we analysed the Person file, focussing on those variables that expressed the properties of habitual commuting patterns [37]. Note that, instead of recording the preferences of respondents in a strict sense, the NHTS database records their behaviour. The advantage of using behavioural data is that we no longer need to take into account the value-action gap often existing in environment-related situations (e.g., [15,38]), hence avoiding the risk of over-estimating environmental-friendly choices in our sample.

Firstly, we identified a set of core variables that could allow us to trace the commuting habits of the respondents who had a job (e.g., person ID, state of residence, number of walk trips, etc.). Secondly, we selected a group of respondents who all answered to the same questions. In other words, we ignored incomplete records to make sure that the data we would work with was consistent. Subsequently, we recoded the values of these variables to normalise the unit measurements and we created three indexes (i.e., one for each eligible modality: M, NM and PT) by computing the simple average between the values of the variables considered for each index. The indexes have then been standardised in order to be used as the initial preferences of our agents [39] and their distribution is presented in Figure 1.

Figure 1. Index distributions: (a) private motorised vehicles (M); (b) private non-motorised vehicles (NM); (c) public transportation (PT).

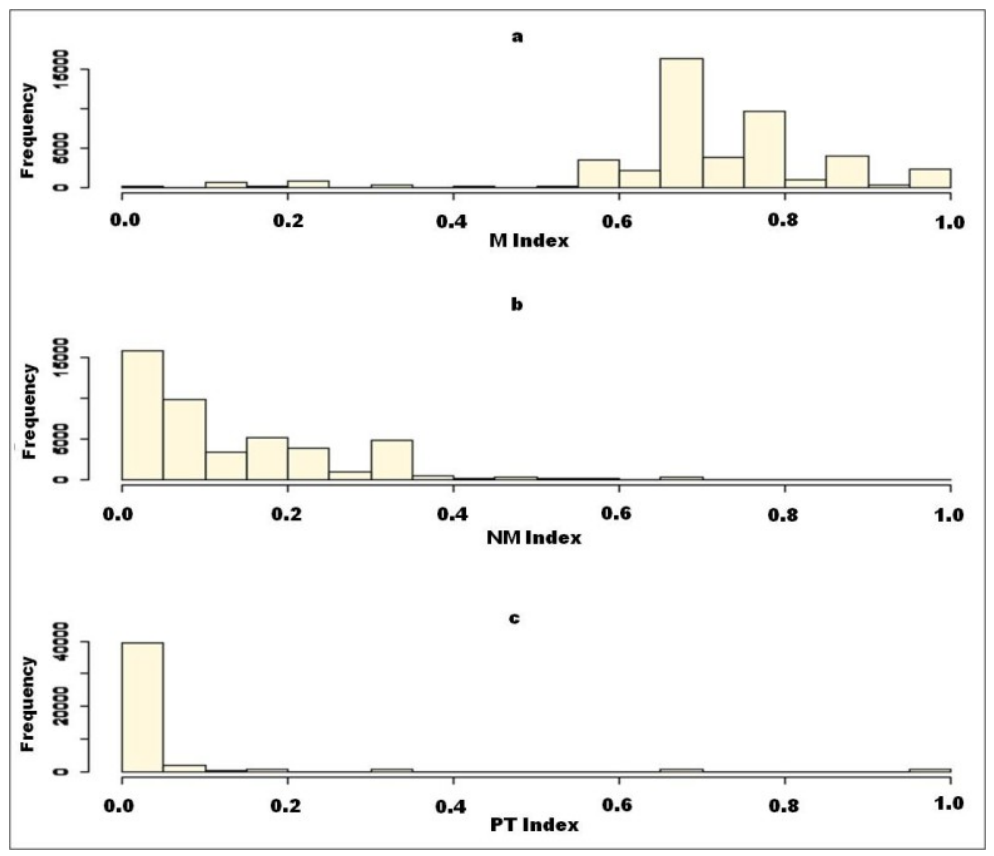




\subsubsection{Other Inputs of MUSA}

Some of the data used by MUSA have been computed separately, in particular the environmental index and the relative cost of each means of transport, which are reported in Table 2. We interrogated the 2009 NHTS once again to find out the average distance from home to work for each type of commute (i.e., SCs and LCs), which resulted in $1.84 \mathrm{mi}$ and $16.25 \mathrm{mi}$, respectively. The environmental index (hereafter $t_{j}$ ) is directly proportional to the emissions produced by each transport modality for an average-length commute, taking as the benchmark the most polluting one. In other words, the emissions of the most polluting means of transport have been divided by the emissions of the transport modality for which the environmental index was being computed and then subtracted from 1. This indicator equals 1 for the "greenest" transportation (i.e., NM) and 0 for the most polluting one (i.e., M). With regard to the relative cost, this has been computed in the same way, although the relation was inverted: the indicator equals 1 for the most expensive transportation and its value decreases accordingly with the expenditure related to the transportation that is being evaluated. The reference values that have been used are summarised in Table 3.

Table 2. Average expenditure and emissions per commute.

\begin{tabular}{cccccc}
\hline Commute & Transportation & $\begin{array}{c}\text { Emissions } \\
\left(\mathbf{K g ~ C O}_{\mathbf{2}}\right)\end{array}$ & Environmental index & $\begin{array}{c}\text { Absolute cost } \\
\text { (\$/mile) }\end{array}$ & Relative cost \\
\hline SC & M & 0.644 & 0.00 & $\$ 1.514$ & 1.00 \\
& NM & 0.000 & 1.00 & $\$ 0.204$ & 0.14 \\
LC & PT & 0.289 & 0.55 & $\$ 0.427$ & 0.28 \\
& M & 5.688 & 0.00 & $\$ 13.373$ & 1.00 \\
\hline
\end{tabular}

Table 3. Data reference for expenditures and emissions.

\begin{tabular}{ccc}
\hline Transportation & Emissions $\left(\mathrm{Kg} \mathrm{CO}_{\mathbf{2}} / \mathbf{m i}\right)$ & Costs $\mathbf{( \$ \mathbf { m i } )}$ \\
\hline $\mathrm{M}$ & 0.350 & $\$ 0.823$ \\
$\mathrm{NM}$ & 0.000 & $\$ 0.111$ \\
$\mathrm{PT}$ & 0.157 & $\$ 0.232$ \\
\hline
\end{tabular}

In Table 3, the reference data for $\mathrm{M}$ emissions $\left(\mathrm{Kg} \mathrm{CO}_{2}\right)$ has been computed as the mean of average emissions for car, light-duty truck and motorcycle, whereas the reference data for PT has been computed as the mean of average emissions for intercity rail, commuter rail, transit rail and bus business travel [40]. The reference data for PT costs per mile has been computed by dividing the 2010 total fares by 2010 total miles covered with PT [41], whereas the reference data for the costs of M has been calculated by computing the mean between the driving costs (i.e., operating costs and ownership costs calculated for 10,000 mi per year) for different private cars (i.e., small sedan, medium sedan, large sedan, 4WD sport utility and minivan) in 2012 [42]. NM costs have been assumed to be $13 \%$ of $\mathrm{M}$ costs. This assumption has been necessary because of the lack of uniform data for the different means of transport. Thus, the value of $13 \%$ value has been computed as the mean between two relative costs of NM in terms of M costs: the first one derives from the report authored by the website 'The Bike Station' [43] and the second one from Litman [44] about Danish Cycling Evaluation. 


\section{Model Calibration}

The calibration of MUSA has been carried out by checking whether the model is able to represent the current distribution of commuting habits in the USA, derived from the TDTD dataset. We varied the need satisfaction $\left(\tau_{n}\right)$ and the uncertainty $\left(\tau_{u}\right)$ thresholds in the $\{0.1,0.2, \ldots, 0.9\}$ set, running 100 repetitions of the model for each parameter combination. The starting point of the model was a situation where everybody used $\mathrm{M}$.

We then chose the parameter combination leading to the average modality distribution - both for SCs and LCs - closest to the one derived from the TDTD database. More specifically, we aggregated the simulation outcomes for each parameter combination keeping SCs and LCs separated, obtaining as a result a single value for each means of transport for each type of commute. Subsequently, we computed the mean squared error (hereafter MSE) between the simulation results and TDTD data for each parameter combination. These are presented in Figure 2.

Figure 2. Overview of calibration results.

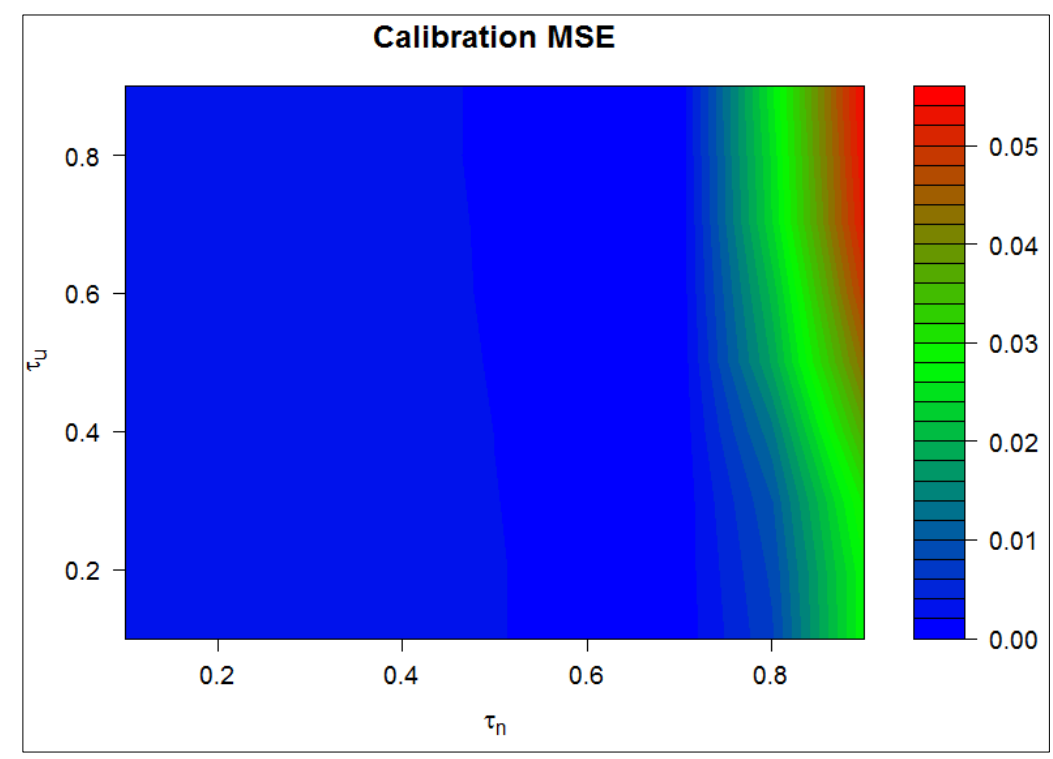

The model was more sensitive to variations in the $\tau_{n}$ parameter, even if $\tau_{u}$ plays a critical role for high levels of $\tau_{n}$ (Figure 2). This was mainly due to the fact that the former parameter crucially discriminates between the two procedures able to produce novel behaviours (social comparison and, especially, rational deliberation) and the two that tend to replicate what is already spread in the population (repetition and imitation). Except in the condition of high $\tau_{n}$, repetition and imitation tend to produce uniform behaviours since all agents are connected with similar ones. Most of the differences between the model outcomes depend on the frequency of the use of the rational deliberation procedure at the beginning of the run, which tends to increase with $\tau_{n}$ but not with $\tau_{u}$. Once the equilibrium is reached, almost all agents instead repeat their previous choices.

The parameter combination that led to the lowest MSE was $\tau_{n}=0.7$ and $\tau_{u}=0.8$. The corresponding MSE was 0.0003 , which is very close to its ideal value (i.e. zero) and led to the following distribution of commuting habits: for SCs, $90 \%$ of the working population commutes by $\mathrm{M}, 3 \%$ by $\mathrm{PT}$ and $7 \%$ by 
NM. As far as LCs are concerned, $96 \%$ of the working population commutes by M, whereas $4 \%$ uses PT. These are presented in Table 4.

Table 4. Difference between empirical and simulated commuting behaviours (proportions).

\begin{tabular}{ccccc}
\hline Commute & Means of transport & Simulated & Empirical & Difference \\
\hline SC & M & 0.896 & 0.886 & 0.010 \\
& PT & 0.030 & 0.170 & 0.013 \\
NM & 0.073 & 0.096 & -0.023 \\
LC & M & 0.963 & 0.979 & -0.017 \\
& PT & 0.037 & 0.017 & 0.020 \\
\hline
\end{tabular}

\section{Results}

This section presents the results of the policy scenarios. In order to investigate the possible behavioural change of the agents corresponding to the implementation of the policies, $\tau_{n}$ and $\tau_{u}$ have been kept fixed at the values given above while changing the price and preference parameters as indicated below.

\subsection{Market-Based Policies}

In this scenario we increased the prices of the modes of transport by $\left(1-t_{j}\right) \delta_{1}$ where $\delta_{1} \in$ $\{0.1,0.2, \ldots, 1.0\}$ represents the policy intensity (i.e., the price increase) and $t_{j}$ is the environmental index of each transport modality. This means that the higher the environmental impact of each modality, the larger the increase in its price, with $\delta_{1}=1$ meaning a doubling of the price of M. A summary of the relative prices of the means of transport corresponding to the policy intensity (i.e., $\delta_{1}$ ) is presented in Table 5. Figure 3 plots the model outcomes for all the $\delta_{1}$ values both for SCs and LCs.

Table 5. Summary of the relative prices of the means of transport corresponding to the market-based policy intensity.

\begin{tabular}{cccccccccccc}
\hline Transportation & $\boldsymbol{\delta}_{\boldsymbol{1}}=\mathbf{0 . 1}$ & $\boldsymbol{\delta}_{\boldsymbol{1}}=\mathbf{0 . 2}$ & $\boldsymbol{\delta}_{\boldsymbol{1}}=\mathbf{0 . 3}$ & $\boldsymbol{\delta}_{\boldsymbol{1}}=\mathbf{0 . 4}$ & $\boldsymbol{\delta}_{\boldsymbol{1}}=\mathbf{0 . 5}$ & $\boldsymbol{\delta}_{\boldsymbol{1}}=\mathbf{0 . 6}$ & $\boldsymbol{\delta}_{\boldsymbol{1}}=\mathbf{0 . 7}$ & $\boldsymbol{\delta}_{\boldsymbol{1}}=\mathbf{0 . 8}$ & $\boldsymbol{\delta}_{\boldsymbol{1}}=\mathbf{0 . 9}$ & $\boldsymbol{\delta}_{\boldsymbol{1}}=\mathbf{1 . 0}$ \\
\hline $\mathbf{M}$ & 1.10 & 1.20 & 1.30 & 1.40 & 1.50 & 1.60 & 1.70 & 1.80 & 1.90 & 2.00 \\
NM & 0.14 & 0.14 & 0.14 & 0.14 & 0.14 & 0.14 & 0.14 & 0.14 & 0.14 & 0.14 \\
PT & 0.33 & 0.37 & 0.42 & 0.46 & 0.51 & 0.55 & 0.60 & 0.64 & 0.69 & 0.73 \\
\hline
\end{tabular}

The implementation of market-based policies led to a significant increase in the share of NM to the detriment of $\mathrm{M}$ for SCs, while PT use remained stable at low levels. Thus for the highest policy intensity $22 \%$ of the agents decided to commute by M, $2 \%$ by PT and $76 \%$ by NM. This configuration led to a $70 \%$ reduction in $\mathrm{CO}_{2}$ emissions from SCs.

On the other hand, market-based policies were unable to provide any large behavioural change in LCs. For the highest policy intensity $95 \%$ of the agents decided to commute by M, whereas $5 \%$ commuted by PT. This configuration led to a mere $0.6 \%$ reduction in $\mathrm{CO}_{2}$ emissions from LCs. 
Figure 3. Market-based policies results for short commutes (SCs) and long commutes (LCs). The red line represents $\mathrm{M}$, the green one represents NM and the blue one represents PT.

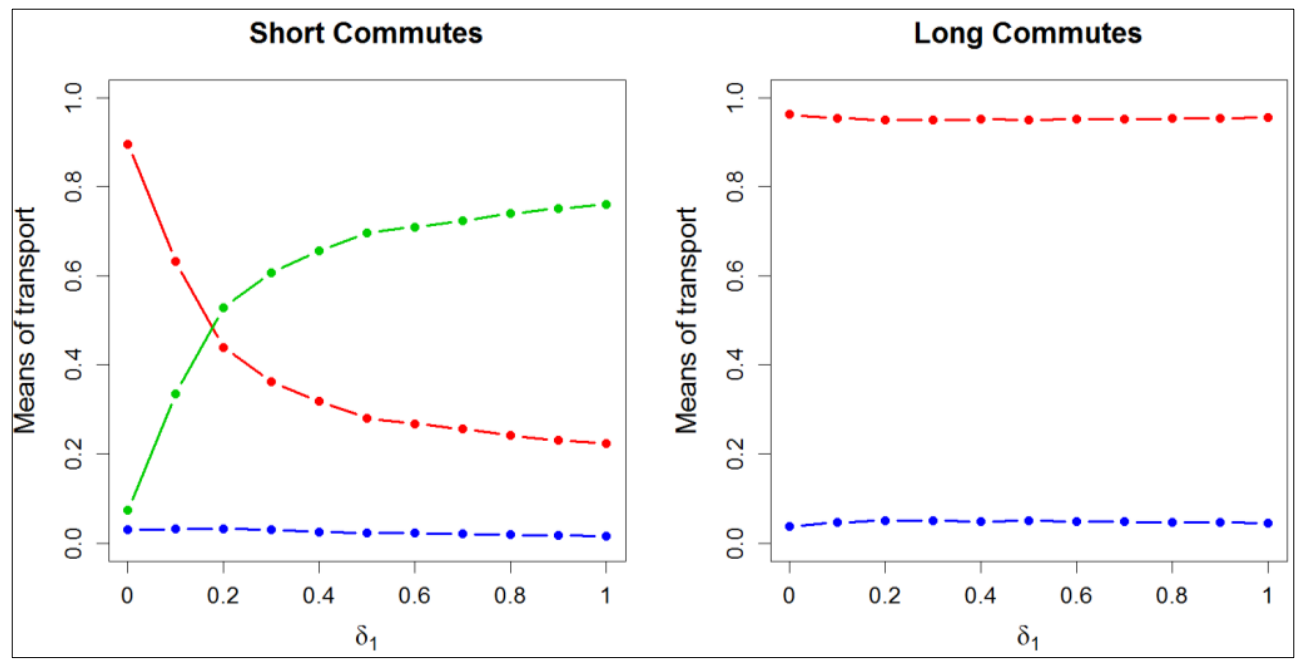

\subsection{Preference-Change Policies}

In this scenario, we tested the implementation of a "green-oriented" combination of policies that could discourage the use of $\mathrm{M}$ while simultaneously favouring the use of less polluting means of transports. These are "preference-change" in the sense that their goal is to change commuters' behaviours starting from their beliefs and concerns. Here, we assumed that all agents are affected equally by these policies.

Practically, preference-change policies affect agents' preferences through a parameter $\delta_{2} \in$ $\{0.1,0.2, \ldots, 1.0\}$ which represents the policy intensity. In this case, the policy intensity is measured as the increase in investments in this type of policy by the relevant stakeholders (e.g., public authorities). Recalling the example given in Section 1, if this policy was actually a combination of policies like marketing campaigns, provision of information about the commuting alternatives of the agents, or the introduction of environmental education throughout the period of formal compulsory education, the policy intensity could be measured as the increase in investments made by the relevant stakeholders (e.g., public authorities) to increase the effectiveness of these policies. This parameter is subtracted from the M preferences, while it is added to the preferences for NM and PT to model a decrease in the willingness to adopt $\mathrm{M}$ transportation and an increase in the willingness to use both NM and PT. The results of the scenarios are presented in Figure 4 both for SCs and LCs.

The implementation of preference-change policies led to a substantial increase in the share of NM to the detriment of M for SCs, while PT share remained almost constant. Since, given the structure of the model, it is trivial that agents with a preference parameter equal to one will always choose the greenest means of transport (Figure 4), we will consider here as actual maximum a policy intensity of $\delta_{2}=0.5$. The modality distribution corresponding to this policy intensity is $4 \%$ of the agents commuted by $\mathrm{M}$, less than $1 \%$ by PT and almost $96 \%$ by NM. This led to a $95 \%$ reduction in $\mathrm{CO}_{2}$ emissions from SCs.

In contrast with market-based policies, preference-change ones led to substantial behaviour change for LCs. The modality distribution corresponding to $\delta_{2}=0.5$ is $4 \%$ of the agents decided to commute by $\mathrm{M}$, whereas $96 \%$ went by PT. This configuration led to a $52 \%$ reduction in $\mathrm{CO}_{2}$ emissions from LCs. 
Figure 4. Preference-change policy results for SCs and LCs. The red line represents M, green $=\mathrm{NM}$ and blue $=\mathrm{PT}$.
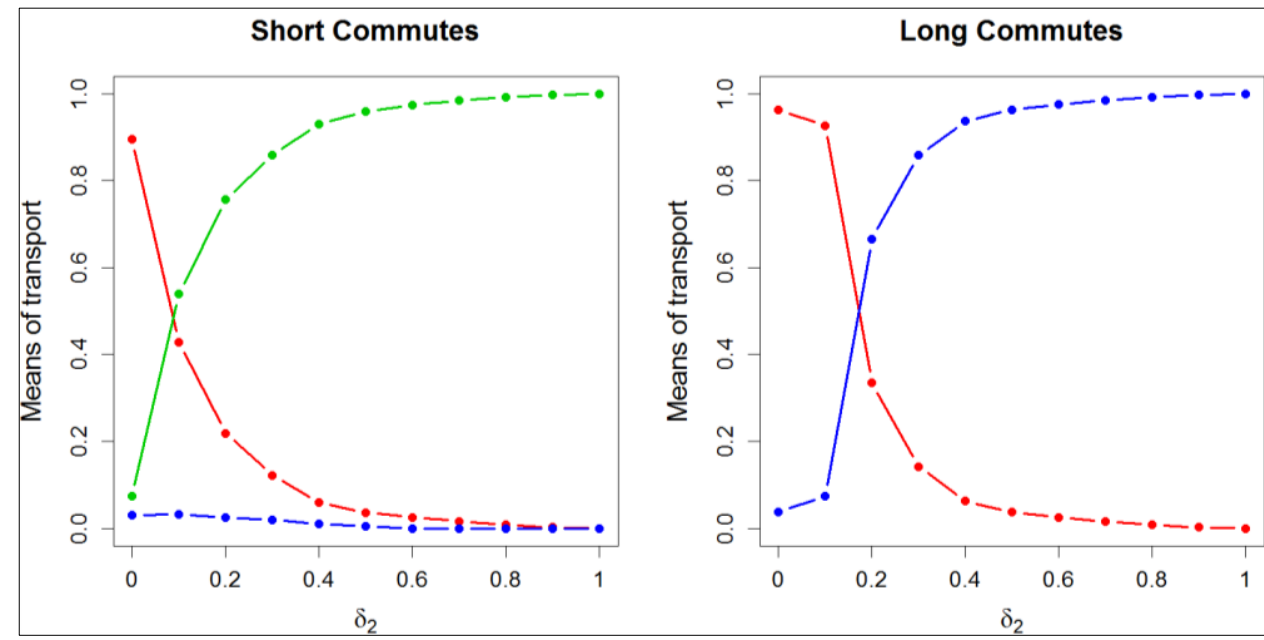

\subsection{Interactions among Policies}

In this scenario we simultaneously implemented both policies (i.e., market-based and preferencechange). The aim of this test was to investigate whether by combining these two policies we could bring about positive synergies, i.e., a change in behaviours at lower policy intensities. The results of this scenario are presented in Figure 5.

Figure 5. Results of the combination of market-based and preference change policies for SCs and LCs. On the axes are the intensities of the policies being implemented and the colour gradient represents the distribution of the agents' behaviours. Dark blue indicates policy combinations that led to $0 \%$ of the agents using that particular means of transport, whereas burgundy represents combinations that led to $100 \%$ of the agents using that particular means of transport.

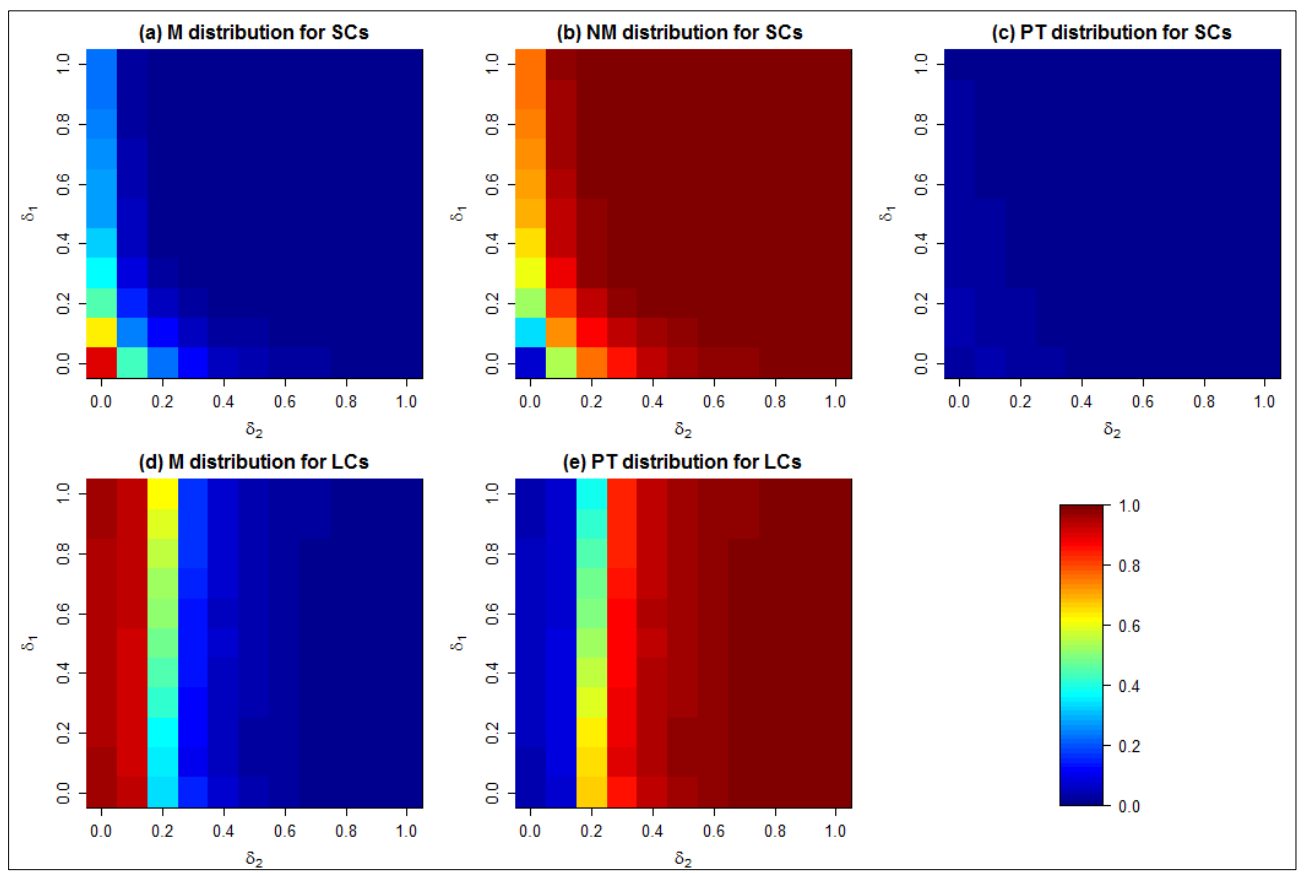


The combination of market- and preference-based policies brings about the switch to NM transportation at lower policy intensities for SCs (Figure 5a-c). Indeed, when market-based policy intensity equals 0.25 and preference-change equals 0.2 (and vice versa) $100 \%$ of the agents commuted by NM. The same does not hold for LCs, where the only policy affecting agents' behaviours is preference-change (Figure 5d,e).

To sum up, the combination of the two policies brings about positive synergies in the SCs but not in the LCs, where the preference-change policies appear to be more effective than market-based ones.

\section{Discussion}

MUSA is an agent-based model developed both to show that this method could be a sound approach to test policy implementation in the transport sector and to analyse the outcomes of the implementation of two sets of policies in the USA's commuting sector. In order to represent the habits of the USA population as realistically as possible, the construction of MUSA followed a multilevel validation, i.e., the initial preferences of the agents have been drawn from the 2009 NHTS and the outputs of the calibration have been checked against different data drawn from the same survey.

The calibration results showed that MUSA was able to reproduce the current commuting habits in the USA. This is partly due to the behavioural richness that has been included in the model by applying the "consumat" framework developed by Jager [31], which gains even more value when coupled with a realistic social network such as the one implemented in our model following the Hamill and Gilbert [34] procedure. These features were critical for both the calibration and policy-testing phases of MUSA.

We tested two types of policies: market-based and preference-change. It is worth noting here that it is not possible to better define the impact of the policies on the overall economy represented in our model. This is because the level of abstraction of MUSA is higher than the different types of policy that can be implemented. Moreover, we assume that the policies being implemented do produce the expected effect, i.e., an increase in the prices of motorised transports and an incentive to prefer non-motorised transports to motorised transports, respectively.

Market-based policies proved to be effective in promoting green behaviours for short commutes. The same cannot be said for long commutes, where the share of private motorised transports remains predominant - and almost unaltered - even after the implementation of the policy. This result is consistent with the existing literature, which often finds transport demand is not price sensitive for long commutes [45].

Preference-change policies, instead, led to the highest share of non-motorised transports and $\mathrm{CO}_{2}$ emissions reduction for both short and long commutes. Nevertheless, the model results suggest that the combination of these policies can be remarkably effective on short commutes, but not on long ones. In the first case their simultaneous implementation creates a positive synergy that is a modal shift to non-motorised transportation at lower policy intensities (Figure 5a-c). This finding appears especially important in terms of policy feasibility since higher effectiveness at lower policy intensities possibly translates into smaller investments to achieve similar results. Conversely, the combination of policies seems to be ineffective for long commutes, where preference-change is the only factor driving the change in agents' behaviour. 
The fact that market-based policies did not significantly affect long commutes resulted in limited benefits in terms of $\mathrm{CO}_{2}$ emission. A doubling of the price of private motorized transport, corresponding to $\delta_{1}=1$, indeed led only to a $0.6 \%$ emission decrease. The same reduction could be achieved by setting $\delta_{2}=0.1$, which probably represents a much easier target in terms of policy feasibility, while higher levels of preference change led to emission reductions up to $52 \%$ for $\delta_{2}=0.5$. Preference-change policies seem therefore to be more effective than price-based ones.

Note also that, in general, the share of public transports is almost never significant for short commutes. This may reflect MUSA's sensitivity to the initial preferences of the agents. As Figure 1c clearly shows, the modal distribution of the public transport index is zero. Hence, this attitude is deeply rooted in American commuters and probably depends on structural motives (e.g., the lack of reliable public transport infrastructures). These cannot be affected by simply increasing the prices of the other modalities - at least under reasonable limits — but only by acting upon the consumers' preferences and opportunities, for instance by significantly improving the current infrastructure level. This is clearly shown by our model. This particular result shows one of the limits of MUSA: the model does not take into account complicating factors such as the diffusion/lack of public transport in the US ground, which might explain the very low preferences among the agents for this means of transport. Other complicating factors might be the presence of adequate infrastructures in general (e.g., cycling and walking lanes, highways, etc.), the income of the commuter and the presence of disabled in the family that can prevent its members to using means of transport in preference to another.

Moreover, the insensitivity of the preferences for public transport is probably reinforced by social influence, represented in MUSA through a social network in which the agents are embedded. This feature had a clear effect in locking the agents into unsustainable behaviours. On the other hand, that behaviours related to short commutes could be easily influenced by policy-making decisions is linked to the much more even preference distribution of the non-motorised transport (see Figure $1 b$ ). Thus, even relatively weak policy intensities could significantly affect the behaviours of the agents that commute for short distances, this time favoured by the social influence effect. Note that both these features are consistent with the dynamics of consumption assumed by Jager [31] and highlighted both in Janssen and Jager [32] and Bravo et al. [33] models.

Notwithstanding the good results obtained by both policies and their combination - at least in short commutes - it is worth noting that economic incentives (i.e., our market-based policies) apparently have to be large to affect travellers' behaviours [46] and that their implementation is likely to bring about only a temporary change in behaviours. Indeed, Dobson [21] found that once these measures are stopped, people's behaviours are likely to revert to those they had before the market-based policy was implemented. It is hence recommended to keep them as a permanent tool to correct market distortions and not just as a temporary incentive. Moreover, Dobson [21] argued that the only way to permanently change behaviours is by changing attitudes, which can be achieved through environmental education and through the formal education system (i.e., our preference-change policies). Indeed, MUSA's results seem to be in line with the idea that preference-change policies are more effective in promoting stable and widespread sustainable behaviours.

Nonetheless, we are aware that convincing people not to use their cars is a real challenge. However, the results related to the implementation of our preference-change policies suggest that if we could find a "trigger policy" that could act on the reason why they prefer to use cars instead of other means of 
transport, their impact could be critical. The formalisation of this kind of policy is a challenge in itself because different communities have different "triggers", but tailoring of policies (e.g., [6,47]) to make them fit closely to the targeted segments of population could bring about successful behaviour change.

In conclusion, two lessons can be learnt from this study. First, the implementation of agent-based modelling in the transport field appears a sound way to test the performances of different policies ex-ante (i.e., before the actual implementation of the policy). This approach needs much lower investments, in terms of both time and money, than ex-post evaluation methods, however depends on the availability and quality of data that is able to inform agents' behaviours and networks to reflect real behaviours. Second, our model based on the data available, shows that the use of different policy interventions are not mutually exclusive. National administrations should therefore use all policy analysis tools available, including agent-based modelling, to test not a single specific policy, but rather the right combination of measures leading to sustainable commuting-styles.

\section{Acknowledgments}

The authors would like to thank Candice Howarth and Robert Evans from the Global Sustainability Institute and three anonymous reviewers for their comments on a previous version of the paper.

\section{Conflicts of Interest}

The authors declare no conflict of interest.

\section{References and Notes}

1. International Energy Agency. $\mathrm{CO}_{2}$ Emissions from Fuel Combustion 2012: Highlights; International Energy Agency Publications: Paris, France, 2012; pp. 1-125.

2. Viceminister of Transport for the Republic of Colombia. Powerpoint Presentation at the 17th Conference of the Parties. In Proceedings of the United Framework Convention on Climate Change, Durban, South Africa, 28 November-9 December 2011.

3. Replogle, M.; Hughes, C. Moving Toward Sustainable Transport. In State of the World 2012: Moving Toward Sustainable Prosperity; Island Press: Washington, DC, USA, 2012; p. 53.

4. International Energy Agency. Transport, Energy and $\mathrm{CO}_{2}-$ Moving Towards Sustainability; International Energy Agency: Paris, France, 2009.

5. Creutzig, F.; McGlynn, E.; Minx, J.; Edenhofer, O. Climate Policies for road transport revisited (I): Evaluation of the current framework. Energ. Policy 2011, 39, 2396-2406.

6. Santos, G.; Behrendt, H.; Teytelboym, A. Part II: Policy Instruments for Sustainable Road Transport. Res. Transport. Econ. 2010, 28, 46-91.

7. Black, W.R. Sustainable transportation: A US perspective. J. Transport. Geogr. 1996, 4, 151-159.

8. Black, W.R. Sustainable Transportation: Problems and Solutions; Guilford Press: New York, NY, USA, 2010.

9. Replogle, M.; Hughes, C. Sustainable Low Carbon Transport: A Weapon against Climate Change. In Future Transport-Cities of the Future; Institute for Transportation and Development Technology (ITDP): New York, NY, USA, 2011; pp. 173-175. 
10. Marsden, G.; Bache, I.; Kelly, C. A Policy Perspective on Transport and Climate Change Issues. In Transport and Climate Change, Transport and Sustainability; Ryley, T., Chapman, L., Eds.; Emerald Group Publishing Limited: Bingley, UK, 2012; Volume 2, pp. 197-396.

11. Ostrom, E.; Janssen, M.A.; Anderies, J.M. Going beyond Panaceas. Proc. Natl. Acad. Sci. USA 2007, 104, 15176-15178.

12. Santos, A.; McGuckin, N.; Nakamoto, H.Y.; Gray, D.; Liss, S. Summary of Travel Trends: 2009 National Household Travel Survey. Available online: http://nhts.ornl.gov/2009/pub/stt.pdf (accessed on 10 June 2012).

13. Buehler, R.; Pucher, J. Making public transport financially sustainable. Transport Policy 2011, 18, 126-138.

14. Flachsland, C.; Brunner, S.; Edenhofer, O.; Creutzig, F. Climate policies for road transport revisited (II): Closing the policy gap with cap-and-trade. Energ. Policy 2011, 39, 2100-2110.

15. Diekmann, A.; Preisendörfer, P. Green and greenback: The behavioral effects of environmental attitudes in low-cost and high-cost situations. Ration. Soc. 2003, 15, 441-472.

16. Diekmann, A.; Preisendörfer, P. Environmental behavior: Discrepancies between aspirations and reality. Ration. Soc. 1998, 10, 79-102.

17. Dunlap, R.E.; McCright, A.M. A widening gap: Republican and democratic views on climate change. Environment 2008, 50, 26-35.

18. Schultz, P.W. Empathizing with nature: The effects of perspective taking on concern for environmental issues. J. Soc. Iss. 2000, 56, 391-406.

19. Young, W.; Hwang, K.; Mcdonald, S.; Oates, C.J. Sustainable consumption: Green consumer behaviour when purchasing products. Sustain. Dev. 2010, 18, 20-31.

20. Jackson, T. Motivating sustainable consumption: A review of evidence on consumer behaviour and behavioural change. Available online: http://www.sd-research.org.uk/wp-content/uploads/ motivatingscfinal_000.pdf (accessed on 13 October 2013).

21. Dobson, A. Environmental citizenship: Towards sustainable development. Sustain. Dev. 2007, 15, 276-285.

22. Anable, J.; Lane, B.; Kelay, T. An evidence based review of public attitudes to climate change and transport behaviour. Available online: http://assets.dft.gov.uk/publications/pgr-sustainablereviewtransportbehaviourclimatechange-pdf/iewofpublicattitudestocl5730.pdf (accessed on 13 October 2013).

23. The MUSA model has been developed using the NetLogo platform (see: Wilensky, U. NetLogo; Center for Connected Learning and Computer-Based Modeling, Northwestern University: Evanston, IL, USA, 1999.) and is available at the following website http://www.openabm.org.

24. Gilbert, N. Agent-Based Models; Sage Publications: Los Angeles, CA, USA, 2008; p. 98.

25. Squazzoni, F. Agent-Based Computational Sociology; Wiley: Chicester, UK, 2012.

26. Gabbriellini, S. Simulare Meccanismi Sociali Con NetLogo. Una Introduzione (in Italian); FrancoAngeli: Milano, Italy, 2011.

27. Grimm, V.; Railsback, S.F. Individual-Based Modeling and Ecology; Princeton University Press: Princeton, NJ, USA, 2005. 
28. Grimm, V.; Berger, U.; Bastiansen, F.; Eliassen, S.; Ginot, V.; Giske, J.; Goss-Custard, J.; Grand, T.; Heinz, S.K.; Huse, G.; et al. A standard protocol for describing individual-based and agent-based models. Ecol. Model. 2006, 198, 115-126.

29. Grimm, V.; Berger, U.; DeAngelis, D.L.; Polhill, J.G.; Giske, J.; Railsback, S.F. The ODD protocol: A review and first update. Ecol. Model. 2010, 221, 2760-2768.

30. Polhill, J.G.; Parker, D.; Brown, D.; Grimm, V. Using the ODD protocol for describing three agent-based social simulation models of land-use change. J. Artif. Soc. Soc. Simulat. 2008, 11, No. 23.

31. Jager, W. Modelling Consumer Behaviour. Ph.D. Dissertation, University of Groningen, The Netherlands, 2000. Available online: http://irs.ub.rug.nl/ppn/240099192 (accessed on 12 October 2013).

32. Janssen, M.A.; Jager, W. Stimulating diffusion of green products: Co-evolution between firms and consumers. J. Evol. Econ. 2002, 12, 283-306.

33. Bravo, G.; Vallino, E.; Cerutti, A.K.; Pairotti, M.B. Alternative scenarios of green consumption in Italy: An empirically grounded model. Environ. Model. Software 2013, 47, 225-234.

34. Hamill, L.; Gilbert, N. Social circles: A simple structure for agent-based social network models. J. Artif. Soc. Soc. Simulat. 2009, 12, No. 3.

35. Barabási, A. Scale-free networks: A decade and beyond. Science 2009, 325, 412-413.

36. U.S. Department of Transportation, Federal Highway Administration. 2009 National Household Travel Survey (Data File and Codebook). Available online: http://nhts.ornl.gov/download.shtml (accessed on 3 May 2012).

37. See the additional materials for a comprehensive list of the selected variables.

38. Flynn, R.; Bellaby, P.; Ricci, M. The 'value-action gap' in public attitudes towards sustainable energy: The case of hydrogen energy. Sociol. Rev. 2009, 57, 159-180.

39. All the statistical analyses were performed using the $\mathrm{R} 3.0$ platform (see: R Core Team. $R: A$ Language and Environment for Statistical Computing; R Foundation for Statistical Computing: Vienna, Austria, 2012. Available online: http://www.R-project.org/ (accessed on 3 May 2012).

40. United States Environmental Protection Agency, Office of Air and Radiation. Optional Emissions from Commuting, Business Travel and Product Transport; United States Environmental Protection Agency: Washington, DC, USA, 2008.

41. National Transport Department. TS 2.2-Service Data and Operating Expenses Time-Series by System (Data File). Available online: http://www.ntdprogram.gov/ntdprogram/data.htm (accessed on 3 May 2012).

42. American Automobile Association. Your Driving Costs: How Much Are You Really Paying to Drive? American Automobile Association: Miami, FL, USA, 2012.

43. The Bike Station. A Better Way to Work: Travel Mode Cost Comparison. Available online: http://www.thebikestation.org.uk/storage/BS_Travel_Cost_Comparison_2011.pdf (accessed on 3 May 2012).

44. Litman, T. Evaluating Non-Motorized Transportation Benefits and Costs; Victoria Transport Policy Institute: Victoria, Canada, 2012.

45. De Jong, G.; Gunn, H. Recent evidence on car cost and time elasticities of travel demand in Europe. J. Transport Econ. Policy 2001, 35, 137-160. 
46. Dargay, J. Personal transport choice. OECD J. 2008, 2, 59-93.

47. Anable, J. 'Complacent car addicts' or 'aspiring environmentalists'? Identifying travel behaviour segments using attitude theory. Transport Policy 2005, 12, 65-78.

(C) 2013 by the authors; licensee MDPI, Basel, Switzerland. This article is an open access article distributed under the terms and conditions of the Creative Commons Attribution license (http://creativecommons.org/licenses/by/3.0/). 